\title{
24 Die Top 10 - damit Ihr Projekt ein Erfolg wird!
}

In diesem Buch haben Sie sicherlich viele Themen gefunden, deren Umsetzung Ihnen helfen wird, Ihr IT-Projekt erfolgreich zu gestalten. Nachfolgend noch einmal die Top 10, die Sie auf jeden Fall berücksichtigen sollten:

1. Strukturieren Sie Ihren Auswahlprozess. Weichen Sie nur in Ausnahmefällen hiervon ab.

2. Definieren Sie Anforderungen (Lastenheft, Pflichtenheft) und Ziele genau und lassen Sie sich nicht vom Anbieter mit tollen Alternativfunktionen ablenken, die Ihren Anforderungen nicht gerecht werden.

3. Wählen Sie nicht einfach einen altbekannten Anbieter oder Marktführer. Recherchieren Sie genau, ob es nicht Alternativen gibt, die einen erheblichen Mehrwert für genau Ihr Unternehmen und Ihre Anforderungen liefern.

4. Ermitteln Sie den Mehrwert der Funktionen und Prozesse beim Einsatz im Unternehmen, um zu erkennen, wo welche Funktionen der neuen Software den meisten Mehrwert liefern sollten.

5. Vermeiden Sie den Fehler der Annahme, wo immer möglich.

6. Glauben Sie dem Anbieter nur das, was er auch bewiesen hat.

7. Motivieren Sie Ihre Mitarbeiter zur Teilnahme und beteiligen Sie diese am Erfolg.

8. Legen Sie einen großen Wert auf Dokumentation, Schulung der Mitarbeiter und ständiges Üben, damit am Go-Live-Tag alles möglichst reibungslos läuft.

9. Verlassen Sie sich nicht auf die Erfahrung der Softwareanbieter. Nutzen Sie eigene Kontrollmechanismen, um Ihr Projekt zu überwachen.

10. Holen Sie sich qualifizierte externe Hilfe. Ihre Kernkompetenz liegt nicht bei der Auswahl und Einführung von IT-Lösungen. 